\title{
Numerical Simulation for a Fractal MIM Model for Solute Transport in Porous Media
}

\author{
Wenyi Liu ${ }^{1}$, Gongsheng $\mathrm{Li}^{1} \&$ Xianzheng Jia ${ }^{1}$ \\ ${ }^{1}$ School of Mathematics and Statistics, Shandong University of Technology, Zibo, China \\ Correspondence: Gongsheng Li, School of Mathematics and Statistics, Shandong University of Technology, Zibo, China
}

Received: March 2, 2021 Accepted: April 3, 2021 Online Published: April 20, 2021

doi:10.5539/jmr.v13n3p31 URL: https://doi.org/10.5539/jmr.v13n3p31

\begin{abstract}
A fractal mobile-immobile (MIM in short) model for solute transport in heterogeneous porous media is investigated from numerics. An implicit finite difference scheme is set forth for solving the coupled system, and stability and convergence of the scheme are proved based on the estimate of the spectral radius of the coefficient matrix. Numerical simulations with different parameters are presented to reveal the solute transport behaviors in the fractal case.
\end{abstract}

Keywords: fractal MIM model, finite difference scheme, stability and convergence, numerical simulation

\section{Introduction}

Solute transport in porous media is a complicated process involving in physical/chemical and biological reactions with fluid mechanics, and the traditional models are the advection-dispersion equations and the MIM solute transport models. The MIM model describes the hydrodynamic behavior in the mobile zone and the mass transfer process between the mobile zone and the immobile zone, which can characterize the physical/chemical non-equilibrium of solute transport in heterogeneous porous media. Although the physical and chemical non-equilibrium models are based on different concepts, they can be described by the same mathematical equation in dimensionless form, see Pang and Close (1999), Toride, Leij and Van Genuchten (1995) for instance. A MIM solute transport undergoing linear sorption without degradations and source/sink reactions in the studied region is given as follows (Benson and Meerschaert (2009), Li, Wen, Zhu and Jakada (2020), Lu, Wang, Zhao and Rathore et al (2018), Schumer and Benson (2003), Van Genuchten and Wagenet (1989)):

$$
\left\{\begin{array}{l}
\beta R \frac{\partial C_{1}}{\partial t}=\frac{1}{P} \frac{\partial^{2} C_{1}}{\partial x^{2}}-\frac{\partial C_{1}}{\partial x}-\omega\left(C_{1}-C_{2}\right), \\
(1-\beta) R \frac{\partial C_{2}}{\partial t}=\omega\left(C_{1}-C_{2}\right),
\end{array}\right.
$$

where $C_{1}, C_{2}$ are the dimensionless solute concentrations in the equilibrium and non-equilibrium sites respectively, $P>0$ is the Pelect number, and $R \geq 1$ is the retardation factor due to the sorption, and $\beta \in(0,1)$ is a partitioning coefficient between the equilibrium and non-equilibrium phases, and $\omega>0$ is the first-order mass transfer rate.

The system (1.1) is a classical integer-order MIM model for solute transport in porous media which has been studied and applied widely by hydrogeologists not only in laboratory but also in field tests. However, there were some researches in the last decades constantly indicated that fractional differential equations could be more suitable than those of classical models to describe non-Darcian flow or anomalous diffusion in some special environment, especially in low-permeability porous media, see Caputo and Plastino (2004), Hansbo (2001), Miller and Low (1963), Obembe, Hossain and Abu-Khamsin (2017), Raghavan (2011), Zhou, Yang and Zhang (2018) for instance. The solute mass transfer or the chemical reaction in a heavy heterogeneous porous media is not an instantaneous process but a longtime dynamical behavior due to the memory effect, in which case fractional diffusion equations incorporating with the memory effect are expected to describe the anomalous diffusion processes in the porous media, see Baeumer and Meerschaert (2007), Benson, Wheatcraft and Meerschaert (2000), Gerolymatou, Vardoulakis and Hilfer (2006), Kelly and Meeschaert (2019), Zhang, Benson and Reeves (2009), Zhou, Yang and Zhang (2019), for instance.

Also by dimensionless we consider a modified model of (1.1) in the unit domain $(0,1)$ for $0<t<T$ by replacing the first-order derivatives on $t>0$ in (1.1) with the corresponding fractional derivatives respectively. Assume that there are the first-order degrading reactions in the solute transportation, and we encounter with the following fractal MIM solute transport system:

$$
\left\{\begin{array}{l}
\beta R_{1} \partial_{t}^{\alpha} u=\frac{1}{P} \frac{\partial^{2} u}{\partial x^{2}}-\frac{\partial u}{\partial x}-\omega(u-v)-\lambda u, \\
(1-\beta) R_{2} \partial_{t}^{\gamma} v=\omega(u-v)-\mu v,
\end{array}\right.
$$

where $u=u(x, t)$ and $v=v(x, t)$ are solute concentrations in the mobile and immobile zones respectively, and $(x, t) \in$ 
$(0,1) \times(0, T) ; R_{1}, R_{2} \geq 1$ are the retardation factors, $\lambda, \mu>0$ are the degradation coefficients in the mobile and immobile respectively; and $P>0$ also denotes the Pelect number, $\beta \in(0,1)$ the partitioning coefficient, and $\omega>0$ the mass transfer rate. In addition, $\partial_{t}^{\alpha}(0<\alpha<1), \partial_{t}^{\gamma}(0<\gamma<1)$ denote the Caputo fractional derivatives on $t>0$, which defined by

$$
\partial_{t}^{\alpha} u(\cdot, t)=\frac{1}{\Gamma(1-\alpha)} \int_{0}^{t} \frac{\partial u(\cdot, s)}{\partial s} \frac{d s}{(t-s)^{\alpha}},
$$

and $\Gamma(\cdot)$ denotes the Gamma function. See the definition of fractional calculus by Kilbas, Srivastava and Trujillo (2006), Podlubny (1999).

The initial value condition is assumed to be

$$
u(x, 0)=0, \quad v(x, 0)=0, \quad 0 \leq x \leq 1,
$$

which means the solute concentration in the mobile and immobile zones are both zero at the initial stage. The boundary condition at $x=0$ is given as

$$
u(0, t)=1, \quad v(0, t)=0, \quad 0<t \leq T,
$$

which implies that the left-hand side of the region in the mobile is a source, and keeps a constant distribution. The condition at $x=1$ is impermeable, which is given by

$$
\frac{\partial u}{\partial x}(1, t)=0, \quad \frac{\partial v}{\partial x}(1, t)=0, \quad 0 \leq t \leq T .
$$

As a result we get a determined system composed by (1.2) with (1.4)-(1.6), which is a novel fractal MIM model for describing reactive solute transport processes with dynamical sub-diffusion behaviors. Its solution can be expressed by the inverse Laplace transform, but the computational cost is very expensive. The finite difference methods are usually utilized for solving fractional differential equations, see Du, Gunzburger, Lehoucq and Zhou (2012), Li, Sun, Jia and Du (2016), Liu, Zhuang, Anh, Turner and Burrage (2007), Liu, Zhuang and Burrage (2014), Meerschaert and Tadjeran (2004), for instance. As for the fractal MIM solute transport system (1.2), there are no studies not only for the solution's properties in mathematics but also for numerical methods. The motivation of this paper is to set forth a numerical method and give numerical simulations for the coupled system. By discretizing the fractional derivatives and integer-order derivatives in (1.2), an implicit finite difference scheme is established, and the unconditional stability and convergence of the scheme are proved by estimating the spectral radius of the coefficient matrix under natural conditions, and numerical simulations are performed with different parameters to reveal the subdiffusion behaviors of the solute in fractal cases.

The rest of the paper is organized as follows.

In section 2, an implicit finite difference scheme for solving the system (1.2) is put forward, and the stability and convergence of the scheme are proved in Section 3, and numerical simulations are presented in Section 4. Concluding remarks are given in section 5 .

\section{The Finite Difference Scheme}

Let $m, n$ be positive integers, and $h=1 / m, \tau=T / n$ be grid steps to discretize the domain. Denote $x_{i}=i h(i=$ $0, \cdots, m), t_{k}=k \tau(k=0, \cdots, n)$ as the grid points, and $u_{1}^{i, k} \approx u\left(x_{i}, t_{k}\right), v^{i, k} \approx v\left(x_{i}, t_{k}\right)$ as the approximations. By the general finite difference method as used to fractional diffusion equations (Li, Sun, Jia and Du (2016), Liu, Zhuang, Anh, Turner and Burrage (2007), Meerschaert and Tadjeran (2004)), we have

$$
\begin{aligned}
& \frac{\beta R_{1}}{\tau^{\alpha} \Gamma(2-\alpha)} \sum_{j=0}^{k}\left[u^{i, j+1}-u^{i, j}\right]\left[(k+1-j)^{1-\alpha}-(k-j)^{1-\alpha}\right] \\
& =\frac{1}{P} \frac{u^{i+1, k+1}-2 u^{i, k+1}+u^{i-1, k+1}}{h^{2}}-\frac{u^{i, k+1}-u^{i-1, k+1}}{h} \\
& -\omega\left(u^{i, k+1}-\frac{v^{i-1, k+1}+v^{i+1, k+1}}{2}\right)-\lambda u^{i, k+1}+O\left(h \tau^{\alpha}+\tau^{\alpha+1}\right),
\end{aligned}
$$

and

$$
\begin{aligned}
& \frac{(1-\beta) R_{2}}{\tau^{\gamma} \Gamma(2-\gamma)} \sum_{j=0}^{k}\left[v^{i, j+1}-v^{i, j}\right]\left[(k+1-j)^{1-\gamma}-(k-j)^{1-\gamma}\right] \\
& =\omega\left(\frac{u^{i-1, k+1}+u^{i+1, k+1}}{2}-v^{i, k+1}\right)-\mu v^{i, k+1}+O\left(\tau^{\gamma+1}\right) .
\end{aligned}
$$

We denote $r_{1}=\frac{\tau^{\alpha} \Gamma(2-\alpha)}{P \beta R_{1} h^{2}}, r_{2}=\frac{\tau^{\gamma} \Gamma(2-\gamma)}{(1-\beta) R_{2}}$, and

$$
\left\{\begin{array}{l}
A=\frac{\tau^{\alpha} \Gamma(2-\alpha)}{\beta R_{1} h}+r_{1}, C=r_{1}, D=\frac{\omega \tau^{\alpha} \Gamma(2-\alpha)}{2 \beta R_{1}}, E=\frac{r_{2} \omega}{2} \\
B=1+A+C+2 D+\frac{\tau^{\alpha} \Gamma(2-\alpha)}{\beta R_{1}} \lambda, F=1+2 E+r_{2} \mu .
\end{array}\right.
$$


By omitting the truncated terms we get an implicit difference equations given as

$$
\left\{\begin{array}{l}
-A u^{i-1, k+1}+B u^{i, k+1}-C u^{i+1, k+1}-D v^{i-1, k+1}-D v^{i+1, k+1} \\
=u^{i, k}-\sum_{j=0}^{k-1}\left(u^{i, j+1}-u^{i, j}\right)\left[(k+1-j)^{1-\alpha}-(k-j)^{1-\alpha}\right], \\
-E u^{i-1, k+1}-E u^{i+1, k+1}+F v^{i, k+1} \\
=v^{i, k}-\sum_{j=0}^{k-1}\left(v^{i, j+1}-v^{i, j}\right)\left[(k+1-j)^{1-\gamma}-(k-j)^{1-\gamma}\right] .
\end{array}\right.
$$

Denote

$$
U^{k}=\left(u^{1, k}, u^{2, k}, \cdots, u^{m-1, k}, v^{1, k}, v^{2, k}, \cdots, v^{m-1, k}\right)^{T}, \quad k=1,2, \cdots, n,
$$

and the initial boundary value conditions are discretized as

$$
\begin{aligned}
U^{(0)} & =\left(u^{1,0}, u^{2,0}, \cdots, u^{m-1,0} ; v^{1,0}, v^{2,0}, \cdots, v^{m-1,0}\right)^{T} \\
& =(0,0, \cdots, 0 ; 0,0, \cdots, 0)^{T},
\end{aligned}
$$

and

$$
\begin{array}{cl}
u^{0, k}=1, \quad v^{0, k}=0, \quad k=0,1, \cdots, n ; \\
u^{m-1, k}=u^{m, k}, \quad v^{m-1, k}=v^{m, k}, \quad k=0,1, \cdots, n .
\end{array}
$$

By rearranging (2.4) we get the difference scheme in the matrix form:

$$
\left\{\begin{array}{l}
M U^{1}=U^{0}, \\
M U^{k+1}=N U^{k}+\sum_{j=1}^{k-1} \Psi_{j}^{k} U^{j}+N_{0} U^{0}, k=1,2, \cdots, n-1,
\end{array}\right.
$$

where the coefficient matrix $M$ is a $2(m-1)$-order matrix defined by

$$
M=\left(\begin{array}{ll}
M_{11} & M_{12} \\
M_{21} & M_{22}
\end{array}\right),
$$

where $M_{11}, M_{12}, M_{21}$ and $M_{22}$ are all $m$ - 1-order matrices given by

$$
\begin{gathered}
M_{11}=\left(\begin{array}{ccccc}
B & -C & 0 & \cdots & 0 \\
-A & B & -C & \cdots & 0 \\
\vdots & \ddots & \ddots & \ddots & \vdots \\
0 & \cdots & -A & B & -C \\
0 & \cdots & 0 & -A & B-C
\end{array}\right), M_{12}=\left(\begin{array}{ccccc}
0 & -D & 0 & \cdots & 0 \\
-D & 0 & -D & \cdots & 0 \\
\vdots & \ddots & \ddots & \ddots & \vdots \\
0 & \cdots & -D & 0 & -D \\
0 & \cdots & 0 & -D & -D
\end{array}\right), \\
M_{21}=\left(\begin{array}{ccccc}
0 & -E & 0 & \cdots & 0 \\
-E & 0 & -E & \cdots & 0 \\
\vdots & \ddots & \ddots & \ddots & \vdots \\
0 & \cdots & -E & 0 & -E \\
0 & \cdots & 0 & -E & -E
\end{array}\right), M_{22}=\left(\begin{array}{ccccc}
F & 0 & 0 & \cdots & 0 \\
0 & F & 0 & \cdots & 0 \\
\vdots & \ddots & \ddots & \ddots & \vdots \\
0 & \cdots & 0 & F & 0 \\
0 & \cdots & 0 & 0 & F
\end{array}\right) .
\end{gathered}
$$

And the matrices $N$ and $N_{0}$ in (2.5) are all 2(m-1)-order defined by

$$
N=\left(\begin{array}{cc}
\left(2-2^{1-\alpha}\right) \mathbf{I} & \mathbf{O} \\
\mathbf{O} & \left(2-2^{1-\gamma}\right) \mathbf{I}
\end{array}\right), N_{0}=\left(\begin{array}{cc}
\xi_{k} \mathbf{I} & \mathbf{O} \\
\mathbf{O} & \zeta_{k} \mathbf{I}
\end{array}\right),
$$

where $\mathbf{I}$ is the $m-1$-order identity matrix, $\mathbf{O}$ denotes the $m-1$-order zero matrix, and

$$
\xi_{k}=(k+1)^{1-\alpha}-k^{1-\alpha}, k=1, \cdots, n-1,
$$

and

$$
\zeta_{k}=(k+1)^{1-\gamma}-k^{1-\gamma}, k=1, \cdots, n-1 .
$$

And the matrix $\Psi_{j}^{k}$ is defined by

$$
\Psi_{j}^{k}=\left(\begin{array}{ll}
b_{1, j}^{k} \mathbf{I} & \mathbf{O} \\
\mathbf{O} & b_{2, j}^{k} \mathbf{I}
\end{array}\right)
$$


where

$$
b_{1, j}^{k}=2(k+1-j)^{1-\alpha}-(k-j)^{1-\alpha}-(k-j+2)^{1-\alpha},
$$

and

$$
b_{2, j}^{k}=2(k+1-j)^{1-\gamma}-(k-j)^{1-\gamma}-(k-j+2)^{1-\gamma},
$$

for $j=1, \cdots, k-1$ and $k=2, \cdots, n-1$.

\section{Stability and Convergence}

According to the background of solute transport in porous media and the physical/chemical laws, the model parameters satisfy the following condition:

$$
0<\gamma, \alpha<1,0<\beta<1, R_{1}, R_{2} \geq 1, P>0, \omega>0, \lambda, \mu>0 .
$$

Under the natural condition (3.1), we will prove the coefficient matrix $M$ given by (2.6) is strictly diagonal dominant, and the finite difference scheme (2.5) is uniquely solvable, and the stability and convergence of the scheme are obtained by estimating the spectral radius of the coefficient matrix.

\subsection{Preliminary Lemmas}

Lemma 3.1 (Jia and $\mathrm{Li}(2016)$ ) For $k=1,2, \cdots$, and $0<\gamma, \alpha<1$, there hold

$$
2 k^{\alpha}-(k-1)^{\alpha}-(k+1)^{\alpha}>0,
$$

and

$$
2 k^{\gamma}-(k-1)^{\gamma}-(k+1)^{\gamma}>0
$$

and the coefficients $b_{1, j}^{k}, b_{2, j}^{k}$ given in (2.11) and (2.12) respectively are all positive.

By Lemma 3.1, we can easily get the following assertions.

Lemma 3.2 For the coefficients $\xi_{k}$, and $\zeta_{k}, k=1,2, \cdots$ defined by (2.8) and (2.9) respectively, there holds

$$
0<\xi_{k}, \zeta_{k}<1 .
$$

Lemma 3.3 Assume $0<\gamma, \alpha<1$ and $k=1,2, \cdots$, there hold

$$
\xi_{k}+\sum_{j=1}^{k-1} b_{1, j}^{k}=2^{1-\alpha}-1,
$$

and

$$
\zeta_{k}+\sum_{j=1}^{k-1} b_{2, j}^{k}=2^{1-\gamma}-1
$$

and there are $b_{1, j}^{k}<1$ and $b_{2, j}^{k}<1$ for $j=1, \cdots, k-1$ and $k=1,2, \cdots$.

The following proposition gives the basis for stability and convergence of the difference scheme (2.5).

Proposition 3.1 For the coefficient matrix $M$ given by (2.6), there holds

$$
M_{i i}>1+\sum_{j=1, j \neq i}^{2 m-2}\left|M_{i j}\right|
$$

with $\sum_{j=1, j \neq i}^{2 m-2} M_{i j}<0$ for $i=1,2, \cdots, 2 m-2$.

Proof Obviously, the coefficients $A, B, C, D, E$, and $F$ defined in (2.3) are all positive under the natural condition (3.1), and the assertion is valid by the following verifications.

(i) $i=1$. Noting $M_{11}=B>0$ and $\sum_{j=2}^{2 m-2} M_{1 j}=-C-D<0$, there is

$$
M_{11}=1+A+C+2 D+\frac{\tau^{\alpha} \Gamma(2-\alpha)}{\beta R_{1}} \lambda>1+\sum_{j=2}^{2 m-2}\left|M_{i j}\right| .
$$

(ii) $i=2, \cdots, m-2$. Noting $M_{i i}=B>0$ and $\sum_{j=1, j \neq i}^{2 m-2} M_{i j}=-A-C-2 D<0$, we also get the assertion (3.7). 
(iii) $i=m-1$. Since $M_{i i}=B-C=1+A+2 D+\frac{\tau^{\alpha} \Gamma(2-\alpha)}{\beta R_{1}} \lambda>0$ and $\sum_{j=1, j \neq m-1}^{2 m-2} M_{m-1} j=-A-2 D<0$. We have

$$
M_{i i}=1+A+2 D+\frac{\tau^{\alpha} \Gamma(2-\alpha)}{\beta R_{1}} \lambda>1+\sum_{j=1, j \neq m-1}^{2 m-2}\left|M_{m-1} j\right|
$$

(iv) $i=m$. Since $M_{m m}=F=1+2 E+r_{2} \mu>0$ and $\sum_{j=1, j \neq m}^{2 m-2} M_{m j}=-E<0$, there holds

$$
M_{m m}=1+2 E+r_{2} \mu>1+\sum_{j=1, j \neq m}^{2 m-2}\left|M_{m j}\right| .
$$

(v) $i=m+1, \cdots, 2 m-2$. Noting $M_{i i}=1+2 E+r_{2} \mu>0$ and $\sum_{j=1, j \neq i}^{2 m-2} M_{i j}=-2 E<0$, follows that the assertion (3.7) is valid too, and the proof is completed.

Corollary 3.1 By this proposition, the coefficient matrix $M$ of the difference scheme (2.5) is strictly diagonal dominant, and the difference scheme is uniquely solvable.

By this proposition we also get an estimates of the spectral radius of the matrix $M$ by direct computations.

Lemma 3.4 For the coefficient matrix $M$ given by (2.6) there hold

$$
1<\rho(M)<2\|M\|_{\infty}-1
$$

and

$$
\frac{1}{2\|M\|_{\infty}-1}<\rho\left(M^{-1}\right)<1,
$$

here $\|M\|_{\infty}=\max _{1<i<2 m-2}\left\{M_{i i}\right\}$, and $\rho(M)$ is the spectral radius of $M$, and there exists a matrix norm $\|\cdot\|_{*}$ such that

$$
\left\|M^{-1}\right\|_{*}<1
$$

where $M^{-1}$ denotes the inverse matrix of $M$.

\subsection{Stability and Convergence}

In this subsection, we discuss the stability and convergence of the difference scheme (2.5) utilizing the norm $\|\cdot\|_{*}$ given in Lemma 3.4, and we denote it as $\|\cdot\|$ for convenience of writing.

Theorem 3.1 Under the natural condition (3.1), the implicit finite difference scheme (2.5) is of unconditional stability.

Proof By the linear difference scheme (2.5), we get

$$
\left\{\begin{array}{l}
M E^{1}=E^{0}, E^{0}=\widetilde{U}^{0}-U^{0}, \\
M E^{k+1}=N E^{k}+\sum_{j=1}^{k-1} \Psi_{j}^{k} E^{j}+N_{0} E^{0},
\end{array}\right.
$$

where $\widetilde{U}^{0}$ denotes the initial function with noises, $E^{k}=\widetilde{U}^{k}-U^{k}$ denotes the solutions difference at the $k$-th level, and $k=0,1, \cdots$.

By (3.11) and Lemma 3.4, there holds

$$
\left\|E^{1}\right\| \leq\left\|M^{-1}\right\|\left\|E^{0}\right\|<\left\|E^{0}\right\| .
$$

Suppose that there are $\left\|E^{j}\right\|<\left\|E^{0}\right\|$ for $j=1,2, \cdots, k$. Then we get also by (3.11) and Lemma 3.4

$$
\begin{aligned}
\left\|E^{k+1}\right\| & \leq\left\|M^{-1}\right\|\left\|N E^{k}+\sum_{j=1}^{k-1} \Psi_{j}^{k} E^{j}+N_{0} E^{0}\right\| \\
& <\left\|N E^{k}+\sum_{j=1}^{k-1} \Psi_{j}^{k} E^{j}+N_{0} E^{0}\right\| \\
& <\left(\|N\|+\sum_{j=1}^{k-1}\left\|\Psi_{j}^{k}\right\|+\left\|N_{0}\right\|\right)\left\|E^{0}\right\| .
\end{aligned}
$$


On the other hand, for given $\alpha, \gamma \in(0,1)$, by Lemmas 3.2-3.3, the sum of norms $\|N\|_{\infty}+\left\|N_{0}\right\|_{\infty}+\sum_{j=1}^{k-1}\left\|\Psi_{j}^{k}\right\|_{\infty}$ is bounded independent of $k \in \mathbf{N}$, and thanks to the equivalence of the norm in finite-dimensional space, there exists a positive constant $C>0$ such that

$$
\left\|E^{k+1}\right\| \leq C\left\|E^{0}\right\|,
$$

which implies that the assertion of this theorem is valid by the inductive principle. The proof is over.

Denote the errors in the solutions by

$$
\begin{aligned}
e^{k}= & \left(u\left(x_{1}, t_{k}\right)-u^{1, k}, u\left(x_{2}, t_{k}\right)-u^{2, k}, \cdots, u\left(x_{m-1}, t_{k}\right)-u^{m-1, k}\right. \\
& \left.v\left(x_{1}, t_{k}\right)-v^{1, k}, v\left(x_{2}, t_{k}\right)-v^{2, k}, \cdots, v\left(x_{m-1}, t_{k}\right)-v^{m-1, k}\right)^{T},
\end{aligned}
$$

for $k=1,2, \cdots, n$, where $u\left(x_{i}, t_{k}\right), v\left(x_{i}, t_{k}\right)$ and $u_{j}^{i, k}, v_{j}^{i, k} j=1,2$, are the exact and numerical solutions at $\left(x_{i}, t_{k}\right)$ of the problem respectively.

Theorem 3.2 The difference solution of (2.5) is convergent to the exact solution as $h, \tau \rightarrow 0$ for any finite time $T<\infty$, and there holds

$$
\left\|e_{k}\right\| \leq \begin{cases}C T^{\gamma}\left(h \tau^{\alpha-\gamma}+\tau+\tau^{1+\alpha-\gamma}\right), & 0<\gamma \leq \alpha<1, \\ C T^{\alpha}\left(h+\tau+\tau^{1+\gamma-\alpha}\right), & 0<\alpha \leq \gamma<1,\end{cases}
$$

where $C$ also denotes positive constant independent of $k \in \mathbf{N}$.

Proof Also by the difference scheme (2.5), and noting to (2.1) and (2.2), we have

$$
\left\{\begin{array}{l}
M e^{1}=R^{1}, \\
M e^{k+1}=N e^{k}+\sum_{j=1}^{k-1} \Psi_{j}^{k} e^{j}+R^{k+1},
\end{array}\right.
$$

where $R^{k}, k=1,2, \cdots$, denote the truncated terms in the solutions' approximation satisfying

$$
\left\|R^{k}\right\| \leq C\left(h \tau^{\alpha}+\tau^{1+\alpha}+\tau^{1+\gamma}\right), k=1,2, \cdots,
$$

here $C>0$ also denotes a positive constant independent of $k \in \mathbf{N}$.

By (3.17) and Lemma 3.4, there holds

$$
\left\|e^{1}\right\| \leq\left\|M^{-1}\right\|\left\|R^{1}\right\| \leq C\left(h \tau^{\alpha}+\tau^{1+\alpha}+\tau^{1+\gamma}\right) .
$$

Assume that

$$
\left\|e^{j}\right\| \leq C\left(h \tau^{\alpha}+\tau^{1+\alpha}+\tau^{1+\gamma}\right), j=1,2, \cdots, k .
$$

We have also by (3.17) and Lemma 3.4

$$
\left\|e^{k+1}\right\| \leq\|N\|\left\|e^{k}\right\|+\sum_{j=1}^{k-1}\left\|\Psi_{j}^{k}\right\|\left\|e^{j}\right\|+\left\|R^{k+1}\right\| .
$$

Consider the case of $0<\gamma \leq \alpha<1$. By Lemma 3.2, and noting that $\xi_{k} \leq \zeta_{k}$ for $0<\gamma \leq \alpha<1$, we have

$$
0<\left\|N_{0}\right\|_{\infty}=\zeta_{k}<1 .
$$

Together with (3.21) and (3.20) and Lemma 3.3, we get

$$
\begin{aligned}
\left\|e^{k+1}\right\| & \leq\left(\|N\|\left\|e^{k}\right\|+\sum_{j=1}^{k-1}\left\|\Psi_{j}^{k}\right\|\left\|e^{j}\right\|+\left\|N_{0}\right\|\left\|R^{k+1}\right\|\right) /\left\|N_{0}\right\| \\
& \leq \frac{C}{\zeta_{k}}\left(\|N\|+\sum_{j=1}^{k-1}\left\|\Psi_{j}^{k}\right\|+\left\|N_{0}\right\|\right)\left(h \tau^{\alpha}+\tau^{1+\alpha}+\tau^{1+\gamma}\right) \\
& \leq \frac{C}{(k+1)^{1-\gamma}-k^{1-\gamma}}\left(h \tau^{\alpha}+\tau^{1+\alpha}+\tau^{1+\gamma}\right) \\
& =\frac{C}{(k+1)^{1-\gamma}-k^{1-\gamma}} \tau^{\gamma}\left(h \tau^{\alpha-\gamma}+\tau+\tau^{1+\alpha-\gamma}\right) .
\end{aligned}
$$




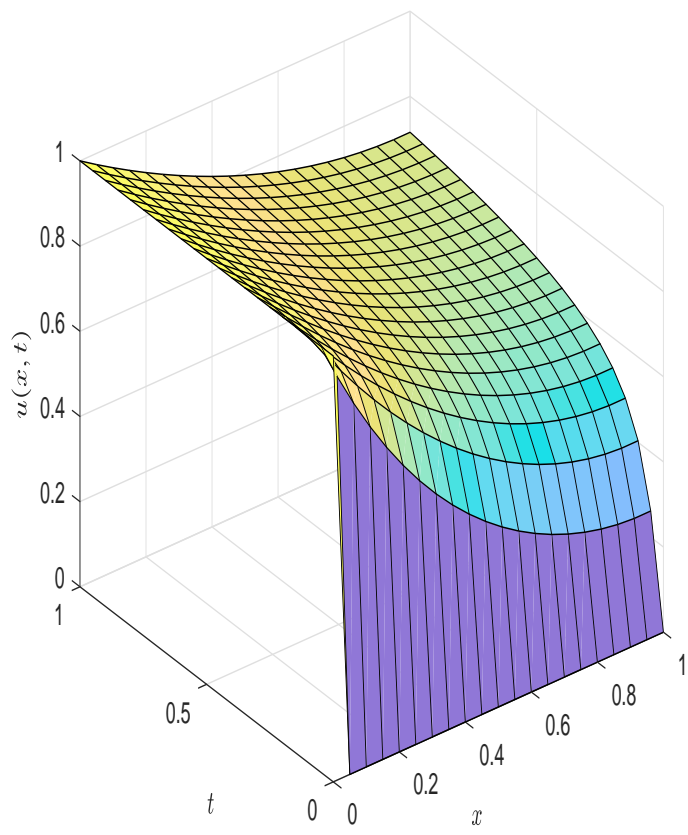

(a) $u$

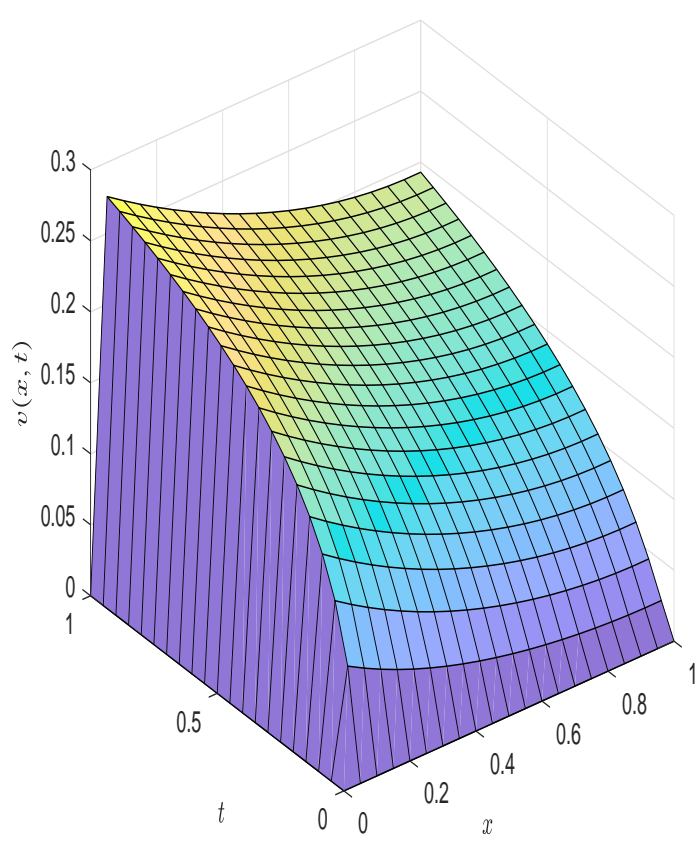

(b) $v$

Figure 1. Numerical solutions of $u$ and $v$ in Ex.1

Noting $\tau=T / n$ and $n \geq k$, we have

$$
\left\|e^{k+1}\right\| \leq \frac{C T^{\gamma}}{\left[(k+1)^{1-\gamma}-k^{1-\gamma}\right] k^{\gamma}}\left(h \tau^{\alpha-\gamma}+\tau+\tau^{1+\alpha-\gamma}\right)
$$

Combining with the limitation

$$
\lim _{k \rightarrow \infty}\left[(k+1)^{1-\gamma}-k^{1-\gamma}\right] k^{\gamma}=1-\gamma,
$$

we get the assertion (3.16) in the case of $0<\gamma \leq \alpha<1$. Similarly we can prove the assertion for $0<\alpha \leq \gamma<1$. This ends the proof of this theorem.

\section{Numerical Tests}

In this section we give numerical simulations using the finite difference scheme (2.5) for the fractal MIM solute transport model, and we will investigate on the transport behaviors by taking different model parameters. It is noted that the computational cost is $O\left(\mathrm{~m}^{3}\right)$ for solving a $m$-order matrix equation, and it becomes $O\left(\mathrm{~m}^{3} \mathrm{n}^{2}\right)$ for the scheme (2.5) for solving the fractal MIM model due to the time-fractional derivatives, where $m, n$ are the numbers of the discretized space and time domains, respectively.

\subsection{Example 1}

The model parameters are chosen as $P=1, \beta=0.5, \omega=0.5, \lambda=0.05, \mu=0.5, R_{1}=R_{2}=2$ and $\alpha=\gamma=0.5$. Taking the space and time steps as $h=1 / 20$ and $\tau=1 / 20$, the finite difference scheme (2.5) is applied to solve the system, and numerical solutions are plotted in Figure 1. In order to observe the solute transport behaviors in the fractal cases, we choose $h=1 / 20, \tau=1 / 100$ and $\alpha=\gamma=0.5$, and the system is solved numerically with different partition parameters and mass transfer rates, and the simulation results are plotted in Figures 2-3.

From Figures 2-3 it can be seen that the partition parameter and the mass transfer rate both have important influences on the solute transport process. The variation of $v$ increases largely and $u$ decreases largely when the partition parameter goes to large. In addition, the mass transfer rate has a great impact on the immobile zone. As the mass transfer rate decreases, the peak value of the variation of $v$ gradually goes down.

Next, we investigate on the dynamics of the system with long time transport. Let $\beta=0.5, \omega=0.5$ and $h=1 / 20, \tau=1 / 20$. By fixing the fractional order in immobile zone be $\gamma=0.5$, and taking the mobile fractional order be $\alpha=0.2,0.4,0.6$ and 


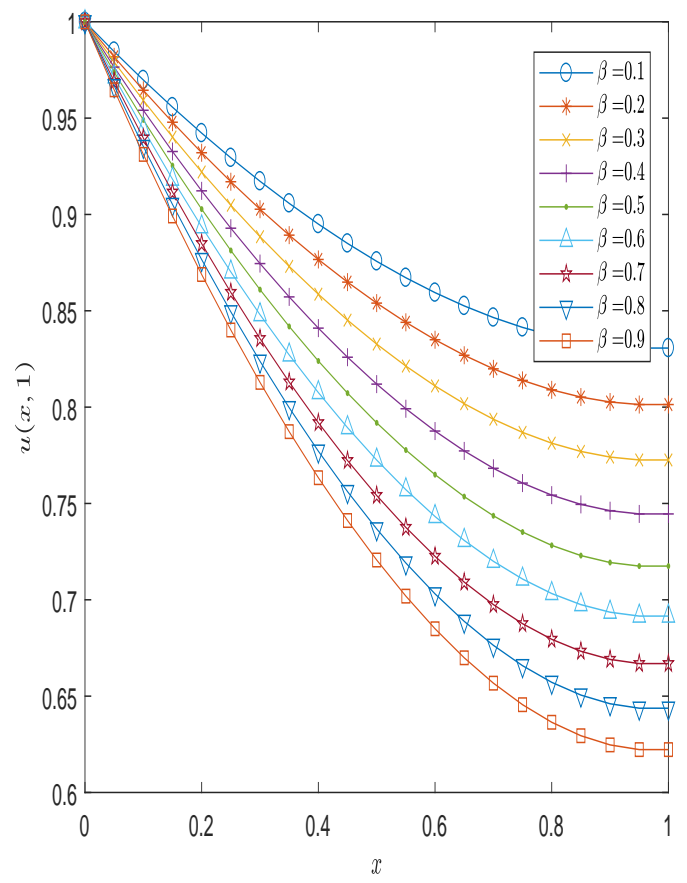

(a) $u$

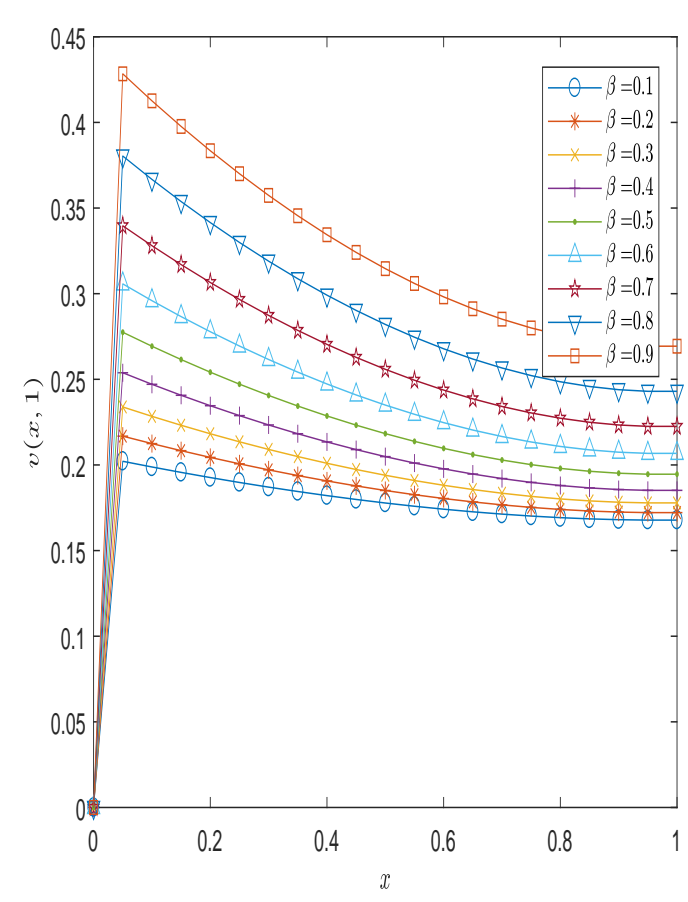

(b) $v$

Figure 2. Transport behaviors with partition parameters $(\omega=0.5)$ in Ex.1

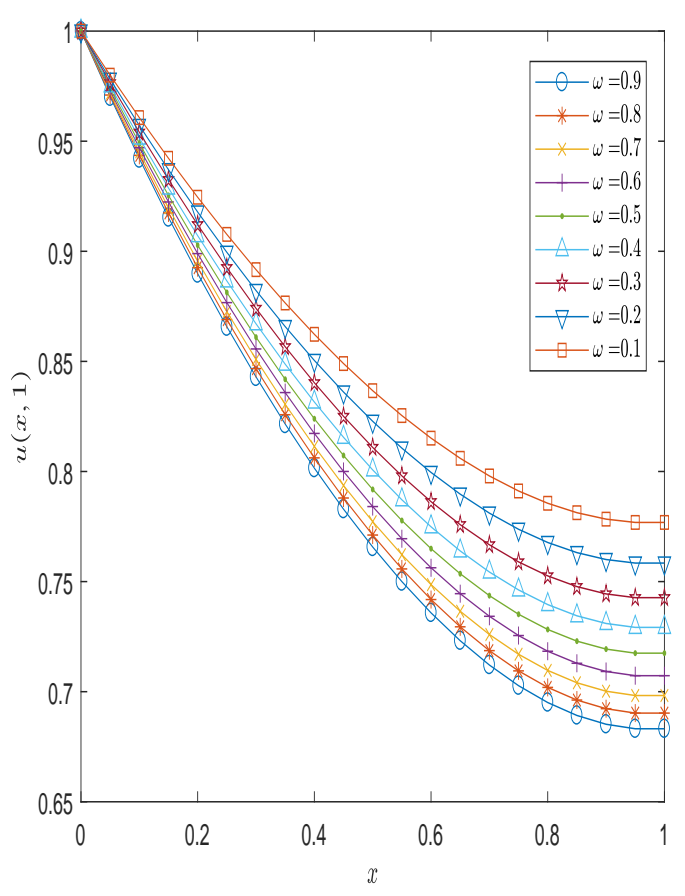

(a) $u$

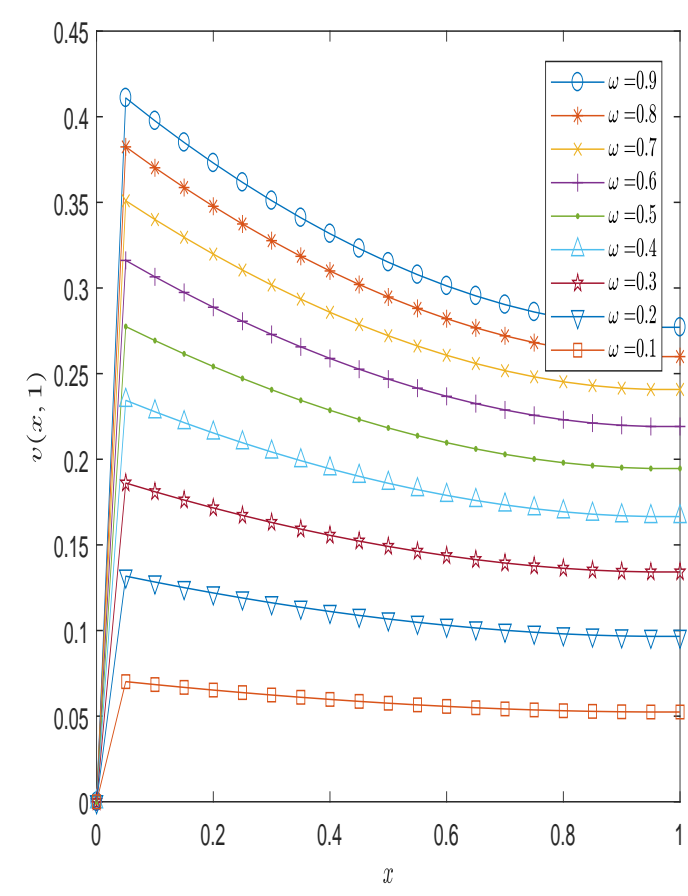

(b) $v$

Figure 3. Transport behaviors with mass transfer rates $(\beta=0.5)$ in Ex.1 


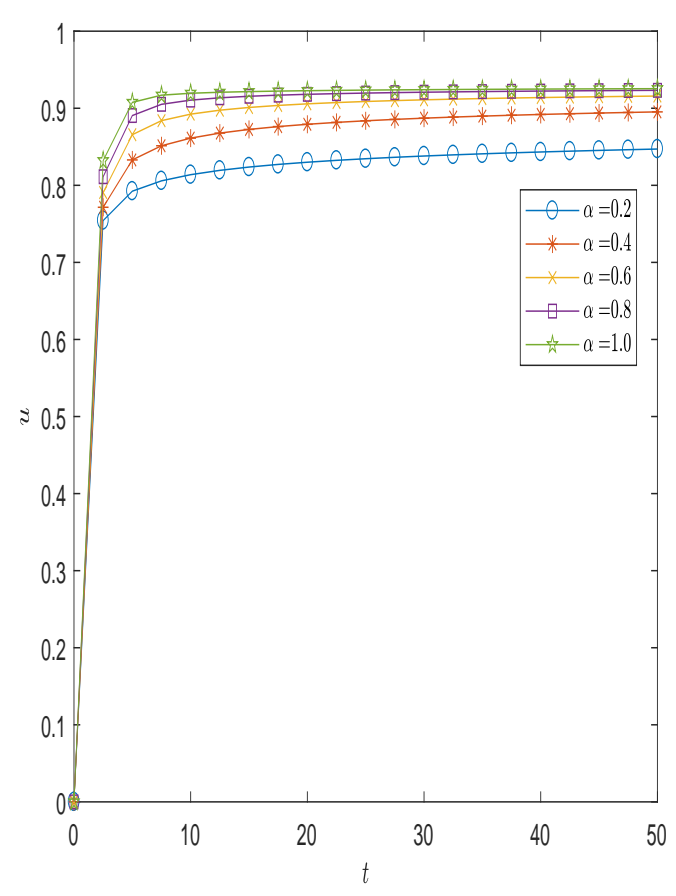

(a) $u$

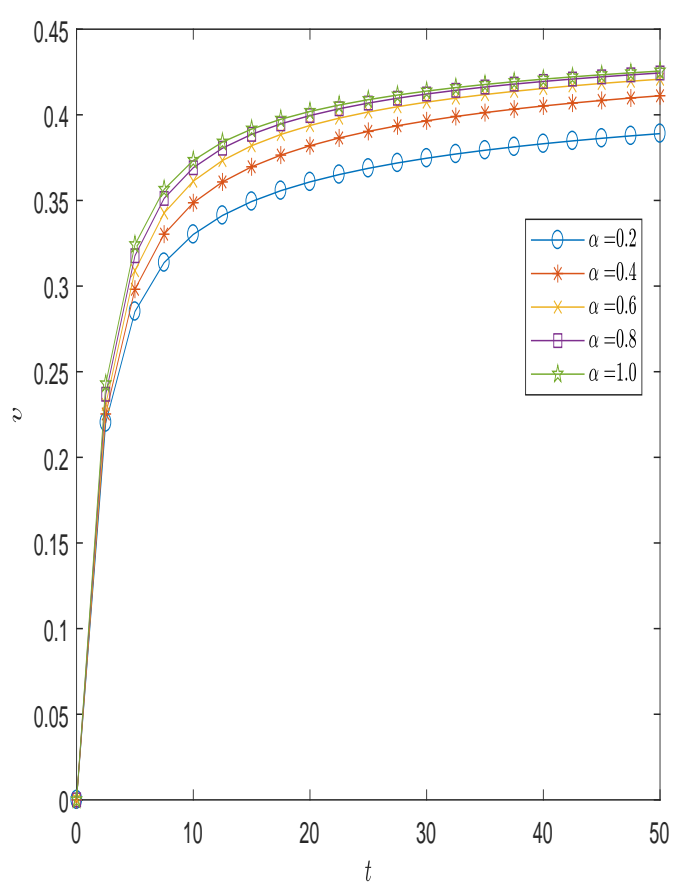

(b) $v$

Figure 4. Long time behaviors of the solute transport with $\gamma=0.5$ in Ex. 1

$\alpha=0.8$ respectively, the solute concentration at $x=0.5$ during the time interval of $[0,50]$ are plotted in Figure 4 , as well as the case of taking $\alpha=1$ where the solute transport in the mobile zone is assumed to be governed by the integer-order diffusion.

From Figure 4 it can be seen that the solute transport becomes slow as the fractional order $\alpha$ goes to small, especially when the transport time $t>5$. It is worth noting that the peak value also decreases as the differential order $\alpha$ decreases. Finally, the solute concentration at $x=0.5$ during the time interval of $[0,50]$ are plotted in Figure 5, where we fix the fractional order in the mobile zone be $\alpha=0.5$, and take the immobile fractional orders be $\gamma=0.2,0.4,0.6$ and $\gamma=0.8$ respectively. As we can see, the immobile fractional order also plays an important role in the solute transport, and the peak value of the solute concentration in the immobile also decreases as the order $\gamma$ becomes small.

\subsection{Example 2}

In this example the model parameters are chosen as $P=1, \beta=0.5, \omega=1.5, \lambda=0.05, \mu=0.1, R_{1}=R_{2}=2$ and $\alpha=0.8, \gamma=0.5$. By using the same time-space steps as done in Ex.1, the numerical solutions are plotted in Figure 6, and the solute transport behaviors are plotted in Figures 7-8 with different partition parameters and mass transfer rates, and the long time behaviors with different fractional orders at $x=0.5$ for $t \in[0,50]$ are plotted in Figures 9-10, respectively.

\section{Conclusion}

A fractal MIM model for reactive solute transport in heterogeneous porous media is investigated from numerics. A stable and convergent finite difference scheme for solving the forward problem is established, and numerical simulations under different conditions are presented. The numerical computations show that the fractal MIM model can be utilized to characterize the subdiffusion behaviors of a solute transport in a long time interval, and the fractional order, the partition parameter and the mass transfer rate all have important influences on the solute transportation. In other words, the fractal transport model is more suitable for describing a long time subdiffusion process, and the solute transport in the immobile zone can not be ignored when coping with heavily heterogeneous porous media. We are to deal with 2D fractal MIM model and focus on inverse problems related with the fractal MIM solute transport models in the near future.

\section{Acknowledgements}

The authors thank the anonymous referees and the editor for their valuable and suggestive comments. This work is supported by National Natural Science Foundation of China (no. 11871313), and Natural Science Foundation of Shandong Province, China (no. ZR2019MA021). 


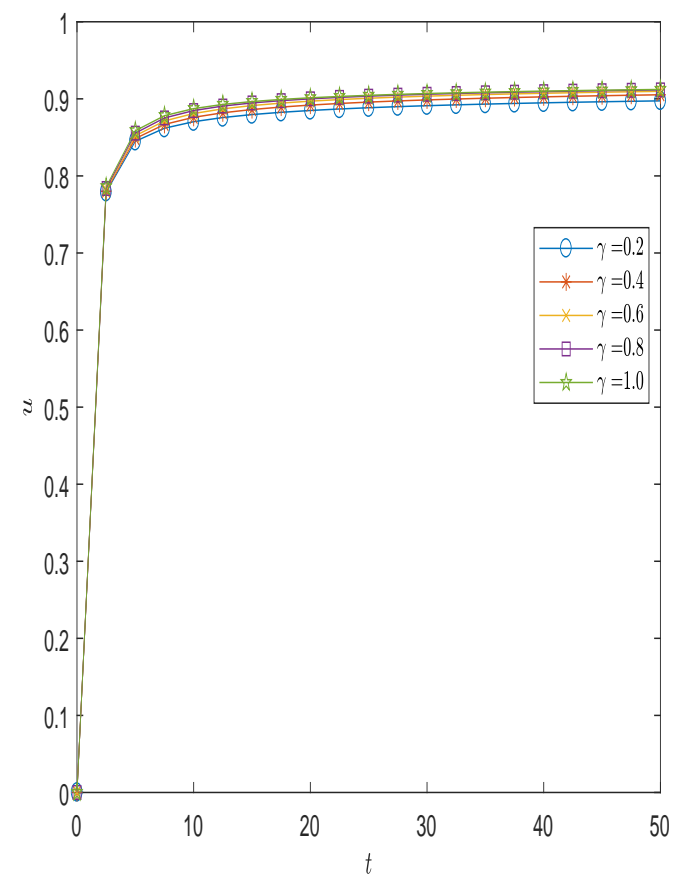

(a) $u$

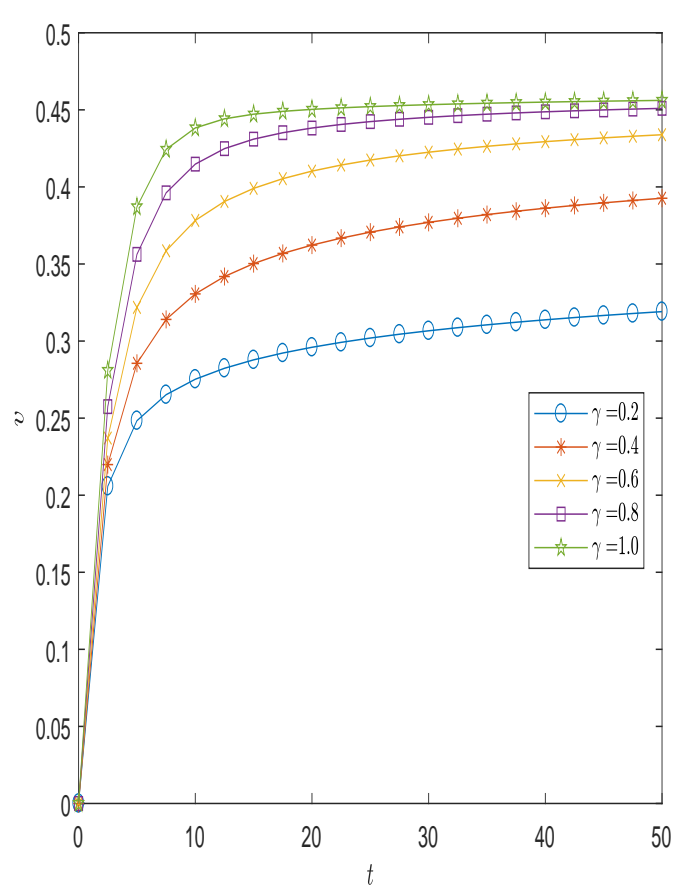

(b) $v$

Figure 5. Long time behaviors of the solute transport with $\alpha=0.5$ in Ex.1

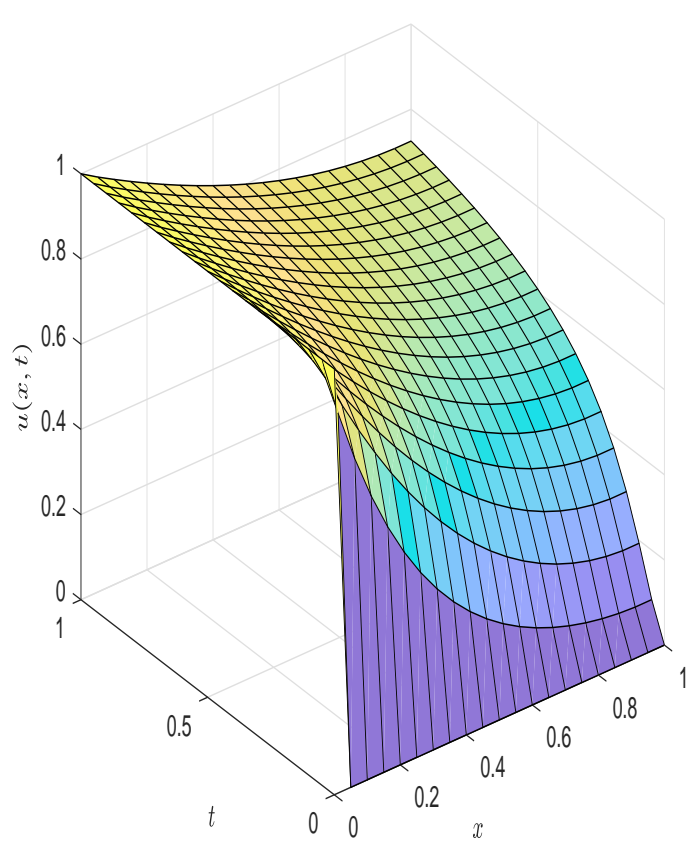

(a) $u$

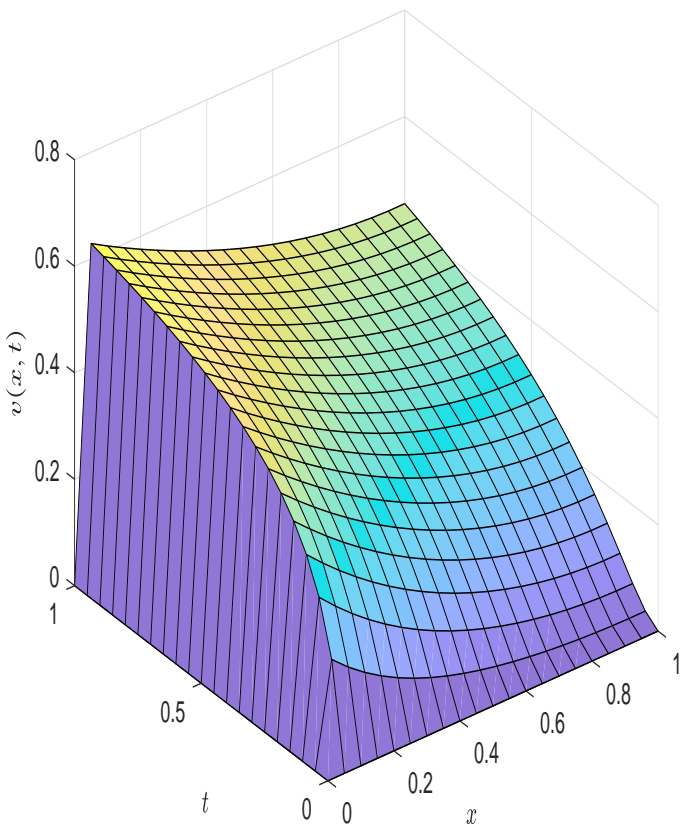

(b) $v$

Figure 6. Numerical solutions of $u$ and $v$ in Ex.2 


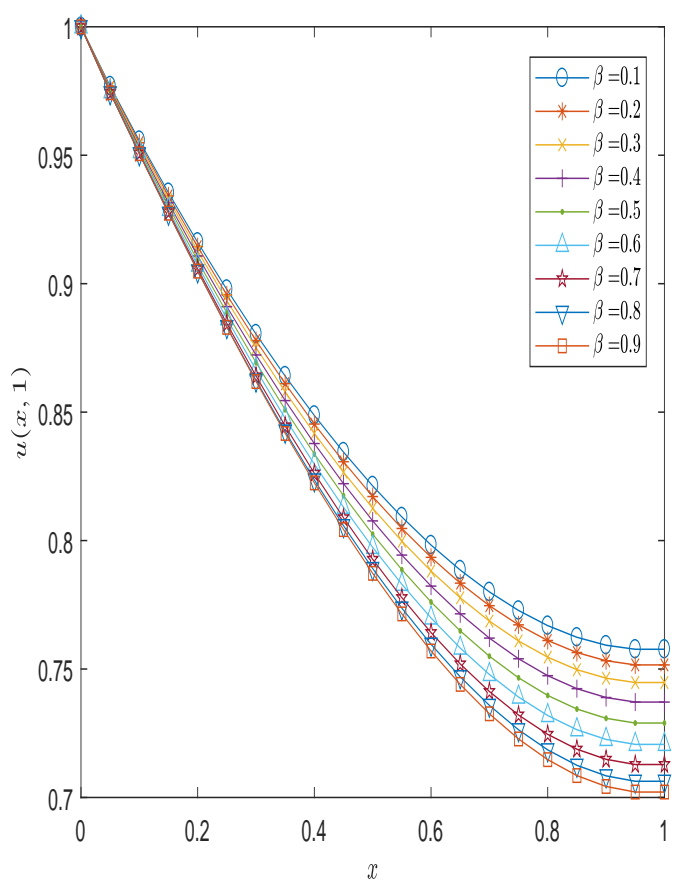

(a) $u$

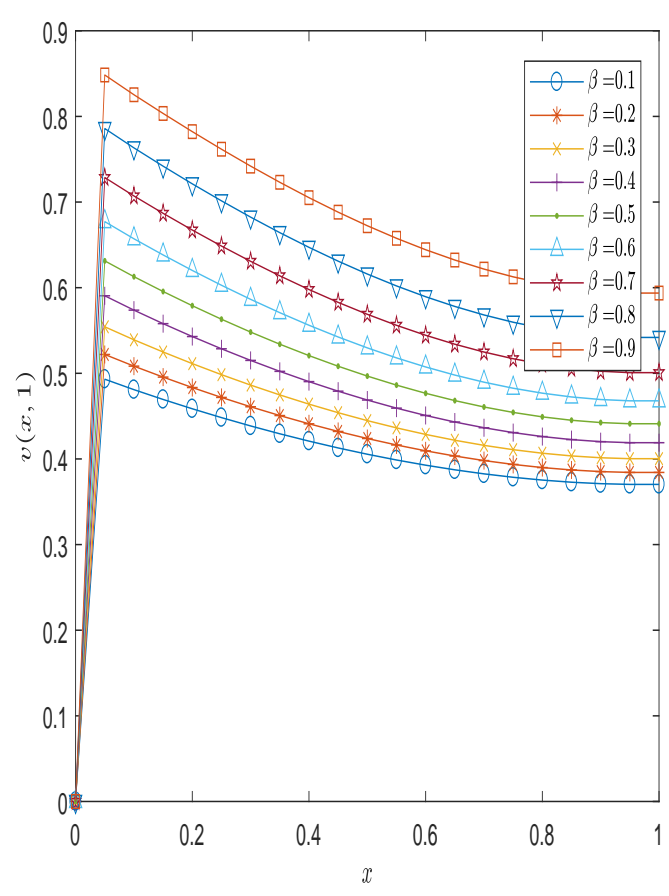

(b) $v$

Figure 7. Transport behaviors with partition parameters $(\omega=1.5)$ in Ex.2

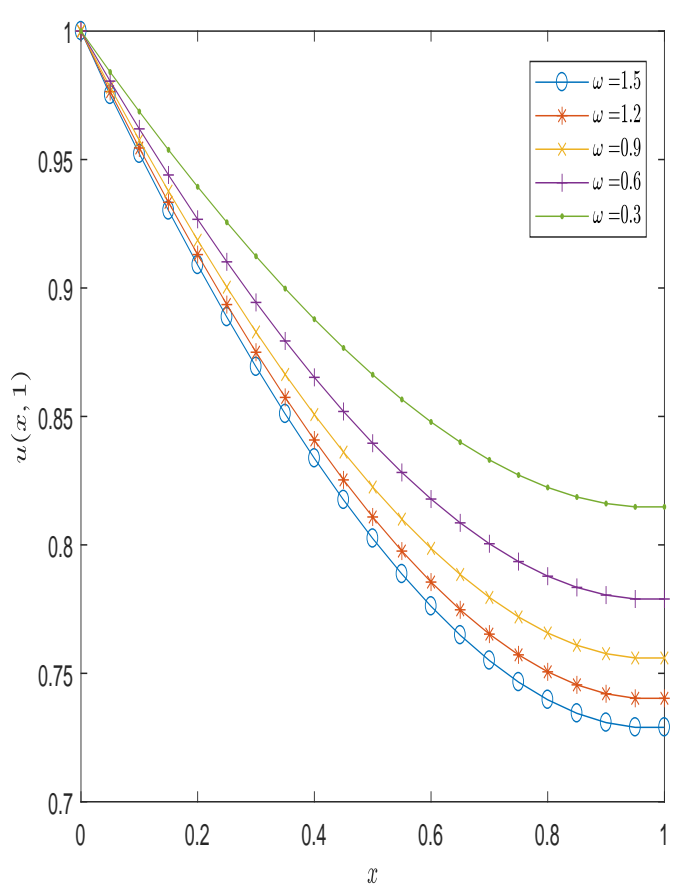

(a) $u$

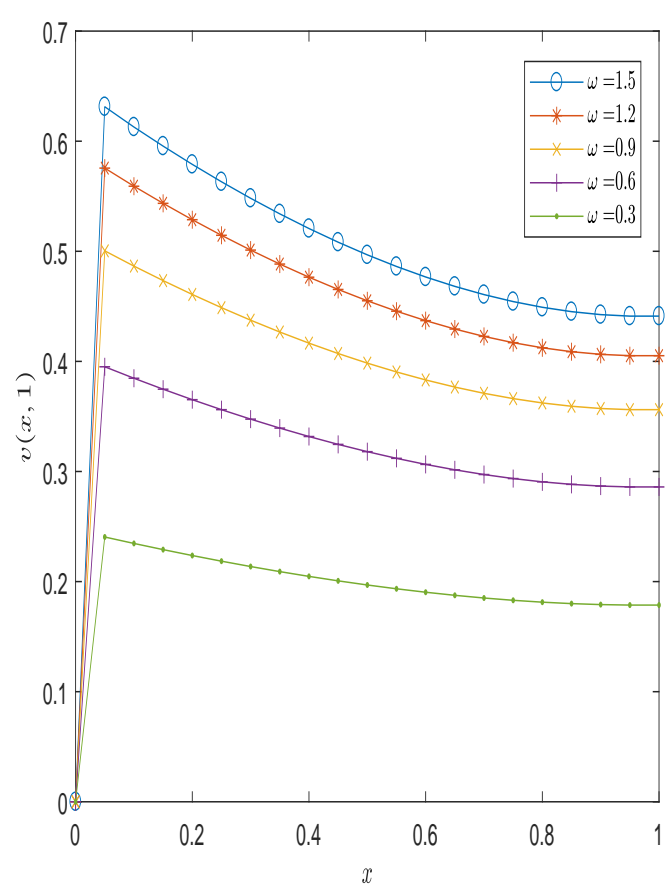

(b) $v$

Figure 8. Transport behaviors with mass transfer rates $(\beta=0.5)$ in Ex.2 


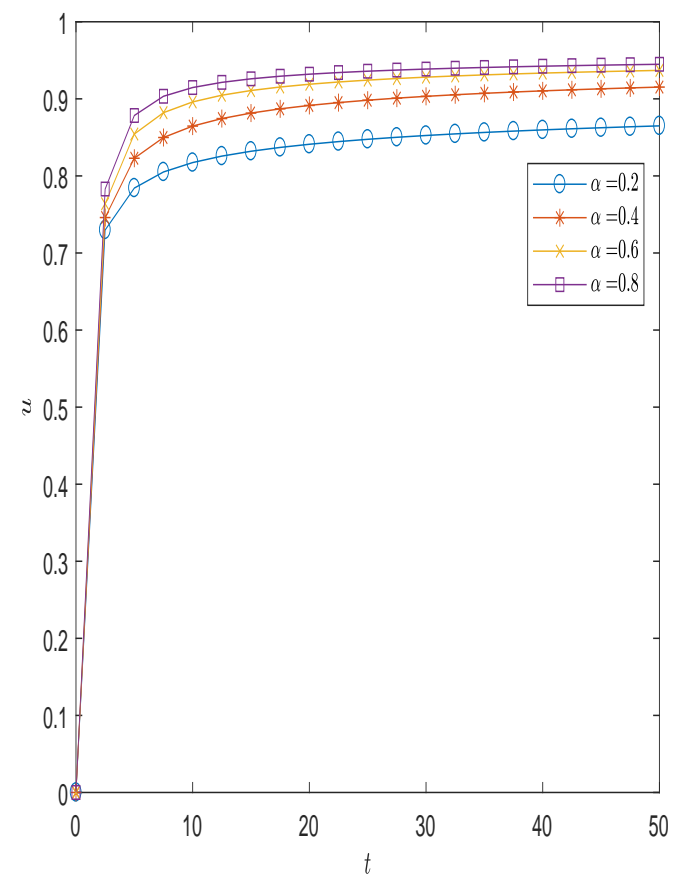

(a) $u$

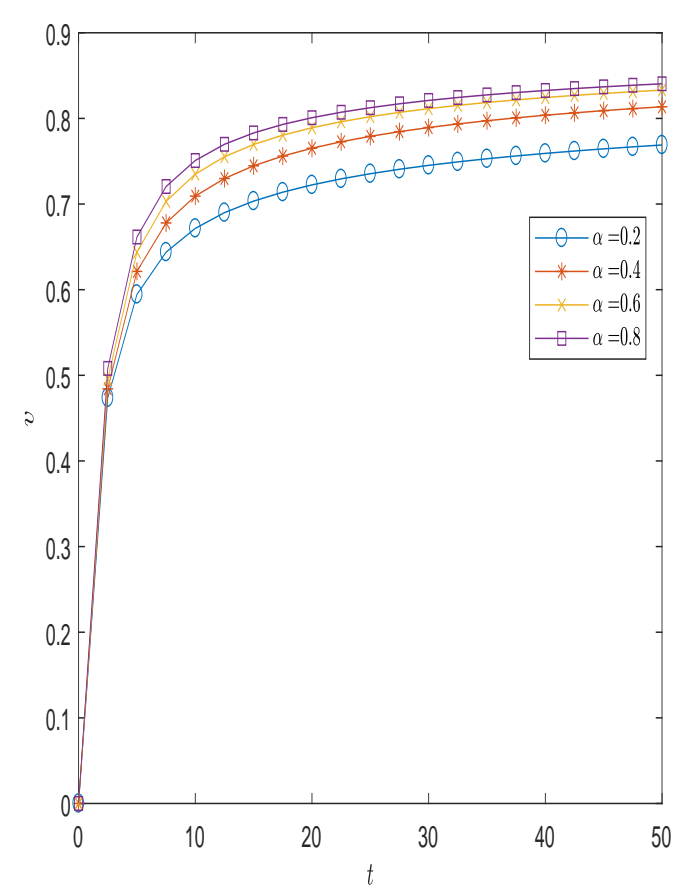

(b) $v$

Figure 9. Long time behaviors of the solute transport with $\gamma=0.5$ in Ex.2

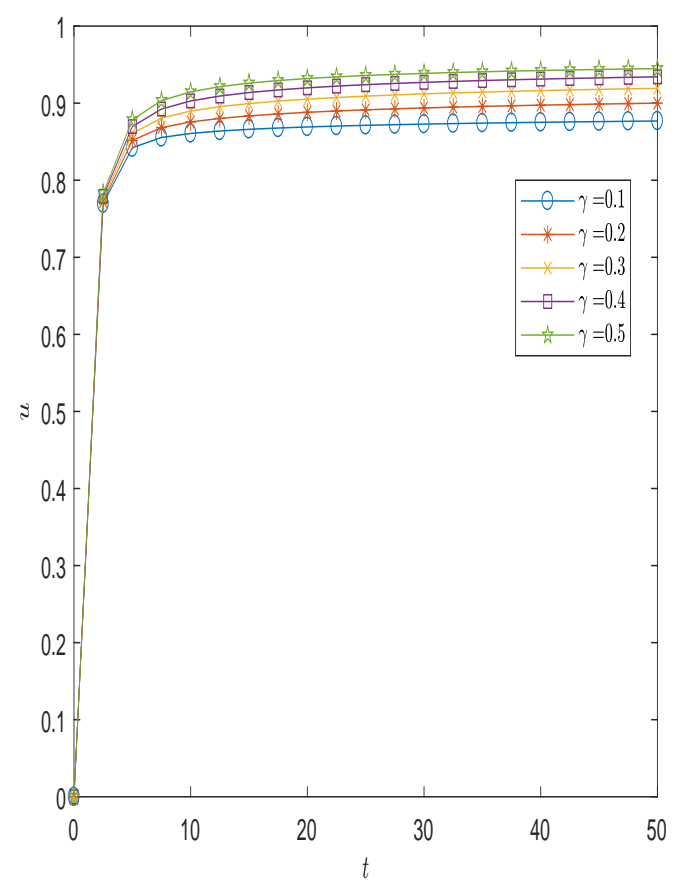

(a) $u$

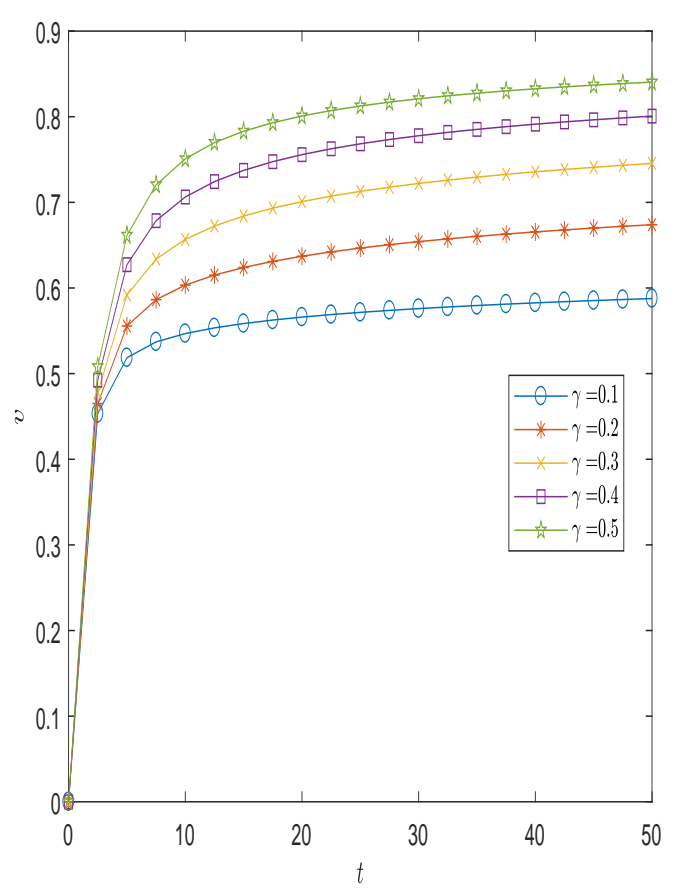

(b) $v$

Figure 10. Long time behaviors of the solute transport with $\alpha=0.8$ in Ex.2 


\section{References}

Baeumer, B., \& Meerschaert, M. M. (2007). Fractional diffusion with two time scales. Physica A: Statistical Mechanics and its Applications, 373, 237-251. https://doi.org/10.1016/j.physa.2006.06.014

Benson, D. A., Wheatcraft, S. W., \& Meerschaert, M. M. (2000). Application of a fractional advection-dispersion equation. Water Resources Research, 36, 1403-1412. https://doi.org/10.1029/2000WR900031

Benson, D. A., \& Meerschaert, M. M. (2009). A simple and efficient random walk solution of multi-rate mobile/immobile mass transport equations. Advances in Water Resources, 32, 532-539. https://doi.org/10.1016/j.advwatres.2009.01.002

Caputo, M., \& Plastino, W. (2004). Diffusion in porous layers with memory. Geophysical Journal International, 158, 385-396. https://doi.org/10.1111/j.1365-246X.2004.02290.x

Du, Q., Gunzburger, M., Lehoucq, K., \& Zhou, K. (2012). Analysis and approximation of nonlocal diffusion problems with volume constraints. SIAM Review, 54, 667-696. https://doi.org/10.1137/110833294

Gerolymatou, E., Vardoulakis, I., \& Hilfer, R. (2006). Modelling infiltration by means of a nonlinear fractional diffusion model. Journal of Physics D: Applied Physics, 39, 4104-4110. https://doi.org/10.1088/0022-3727/39/18/022

Hansbo, S. (2001). Consolidation equation valid for both Darcian and non-Darcian flow. Geotechnique, 51, 51-54. https://doi.org/10.1680/geot.2001.51.1.51

Jia, X. Z., \& Li, G. S. (2016). Numerical simulation and parameters inversion for non-symmetric two-sided fractional advection-dispersion equation. Journal of Inverse and Ill-Posed Problems, 24, 29-39. https://doi.org/10.1515/jiip2013-0040

Kelly, J. F., \& Meeschaert, M. M. (2019). Space-time duality and high-order fractional diffusion. Physical Review E, 99, 022122. https://doi.org/10.1103/PhysRevE.99.022122

Kilbas, A. A., Srivastava, H. M., \& Trujillo, J. J. (2006). Theory and Applications of Fractional Differential Equations. Amsterdam: Elsevier.

Li, G. S., Sun, C. L., Jia, X. Z., \& Du, D. H. (2016). Numerical solution to the multi-term time fractional diffusion equation in a finite domain. Numerical Mathematics: Theory, Methods and Applications, 9, 337-357. https://doi.org/10.4208/nmtma.2016.y13024

Li, X., Wen, Z., Zhu, Q., \& Jakada, H. (2020). A mobile-immobile model for reactive solute transport in a radial two-zone confined aquifer. Journal of Hydrology, 580, 124347. https://doi.org/10.1016/j.jhydrol.2019.124347

Liu, F., Zhuang, P., Anh, V., Turner, I., \& Burrage, K. (2007). Stability and convergence of the difference methods for the space-time fractional advection-diffusion equation. Applied Mathematics and Computation, 191, 12-20. https://doi.org/10.1016/j.amc.2006.08.162

Liu, F., Zhuang, P., \& Burrage, K. (2014). A new fractional finite volume method for solving the fractional diffusion equation. Applied Mathematical Modelling, 38, 3871-3878. https://doi.org/10.1016/j.apm.2013.10.007

Lu, C., Wang, Z., Zhao, Y., \& Rathore, S. S., et al. (2018). A mobile-immobile solute transport model for simulating reactive transport in connected heterogeneous fields. Journal of Hydrology, 560, 97-108. https://doi.org/10.1016/j.jhydrol.2018.02.073

Meerschaert, M. M., \& Tadjeran, C. (2004). Finite difference approximations for fractional advection-dispersion flow equations. Journal of Computational and Applied Mathematics, 172, 65-77. https://doi.org/10.1016/j.cam.2004.01.033

Miller, R. J., \& Low, P. F. (1963). Threshold gradient for water flow in clay systems. Soil Science Society of America Journal, 27, 605-609. https://doi.org/10.2136/sssaj1963.03615995002700060013x

Obembe, A. D., Hossain, M. E., \& Abu-Khamsin, S. A. (2017). Variable-order derivative time fractional diffusion model for heterogeneous porous media. Journal of Petroleum Science and Engineering, 152, 391-405. https://doi.org/10.1016/j.petrol.2017.03.015

Pang, L. P., \& Close, M. E. (1999). Non-equilibrium transport of Cd in alluvial gravels. Journal of Contaminant Hydrology, 36, 185-206. https://doi.org/10.1016/10.1016/S0169-7722(98)00110-7

Podlubny, I. (1999). Fractional Differential Equations. San Diego: Academic Press. 
Raghavan, R. (2011). Fractional derivatives: application to transient flow. Journal of Petroleum Science and Engineering, 80, 7-13. https://doi.org/10.1016/j.petrol.2011.10.003

Schumer, R., \& Benson, D. A. (2003). Fractal mobile/immobile solute transport. Water Resources Research, 39, 12961308. https://doi.org/10.1029/2003WR002141

Toride, N., Leij, F. J., \& Van Genuchten, M. T. (1995). The CXTFIT Code for Estimating Transport Parameters from Laboratory or Field Tracer Experiments, Version 2.0, U. S. Department of Agriculture. Research Report, 137.

Van Genuchten, M. T., \& Wagenet, R. J. (1989). Two-site/two-region models for pesticide transport and degradation: Theoretical development and analytical solutions. Soil Science Society of America Journal, 53, 1303-1310. https://doi.org/10.2136/sssaj1989.03615995005300050001x

Zhang, Y., Benson, D. A., \& Reeves, D. M. (2009). Time and space nonlocalities underlying fractional-derivative models: Distinction and literature review of field applications. Advances in Water Resources, 32, 561-581. https://doi.org/10.1016/j.advwatres.2009.01.008

Zhou, H. W., Yang, S., \& Zhang, S. Q. (2018). Conformable derivative approach to anomalous diffusion. Physica A: Statistical Mechanics and its Applications, 491, 1001-1013. https://doi.org/10.1016/j.physa.2017.09.101

Zhou, H. W., Yang, S., \& Zhang, S. Q. (2019). Modeling non-Darcian flow and solute transport in porous media with the Caputo-Fabrizio derivative. Applied Mathematical Modelling, 68, 603-615. https://doi.org/10.1016/j.apm.2018.09.042

\section{Copyrights}

Copyright for this article is retained by the author(s), with first publication rights granted to the journal.

This is an open-access article distributed under the terms and conditions of the Creative Commons Attribution license (http://creativecommons.org/licenses/by/4.0/). 